\title{
Regime Transition in Electromechanical Fluid Atomization and Implications to Analyte Ionization for Mass Spectrometric Analysis
}

\author{
Thomas P. Forbes, ${ }^{a}$ F. Levent Degertekin, ${ }^{\mathrm{a}, \mathrm{b}}$ and Andrei G. Fedorov ${ }^{\mathrm{a}, \mathrm{b}}$ \\ ${ }^{a}$ G. W. Woodruff School of Mechanical Engineering, Georgia Institute of Technology, Atlanta, Georgia, USA \\ ${ }^{b}$ Parker H. Petit Institute for Bioengineering and Bioscience, Georgia Institute of Technology, Atlanta, \\ Georgia, USA
}

\begin{abstract}
The physical processes governing the transition from purely mechanical ejection to electromechanical ejection to electrospraying are investigated through complementary scaling analysis and optical visualization. Experimental characterization and visualization are performed with the ultrasonically-driven array of micromachined ultrasonic electrospray (AMUSE) ion source to decouple the electrical and mechanical fields. A new dimensionless parameter, the Fenn number, is introduced to define a transition between the spray regimes, in terms of its dependence on the characteristic Strouhal number for the ejection process. A fundamental relationship between the Fenn and Strouhal numbers is theoretically derived and confirmed experimentally in spraying liquid electrolytes of different ionic strength subjected to a varying magnitude electric field. This relationship and the basic understanding of the charged droplet generation physics have direct implications on the optimal ionization efficiency and mass spectrometric response for different types of analytes. (J Am Soc Mass Spectrom 2010, 21, 1900-1905) (C) 2010 Published by Elsevier Inc. on behalf of American Society for Mass Spectrometry
\end{abstract}

$\mathrm{R}$ egime transition in spray formation and understanding the basic relationships between spray modes (e.g., jet versus droplet ejection) and fluid properties are of fundamental scientific interest and broad practical importance. Early work can be traced back to Lord Rayleigh's original investigations of jet breakup through a linear stability analysis [1-3]. Over the years, studies of different modes of transition and stability under various environmental conditions have appeared in the literature, including jet atomization [4-5], dripping [6-8], and ultrasonic ejection [9]. One area that has gained a great deal of interest is liquid jetting and droplet formation under the application of an electric field. Investigations in this area date back to the pioneering works of Zeleny [10] and Taylor [11]. More recent works on fluid physics in the presence of an electric field include studies of the transitions in drop formation [12], jet instability and breakup [13, 14], electrohydrodynamic tip streaming [15], and transitions in electrospraying [16]. Previously, regime transitions have been analyzed for isolated cases of either purely mechanically-driven or electrically-driven atomization. In this paper, we investigate a case of coupled electromechanical phenomena in spraying electrolytes under the application of an external electric field, aiming to develop the fundamental scaling relationship to define the transition between ejection regimes.

Address reprint requests to Professor A. G. Fedorov, G. W. Woodruff School of Mechanical Engineering, Georgia Institute of Technology, Atlanta, GA, USA. E-mail: andrei.fedorov@me.gatech.edu
The ultrasonically-driven array of micromachined ultrasonic electrospray (AMUSE) ion source for mass spectrometry (MS) is used as a unique analytical tool to study the regime transition by virtue of AMUSE's capability to individually control the electrical and mechanical fields driving droplet ejection and charge separation processes $[9,17-23]$. Identification of various spraying regimes and physical understanding of the ejection processes play an essential role in maximizing ionization efficiency of analytes with different physico-chemical properties, leading to improvements in mass spectrometric signal abundance, sensitivity, and stability. Here, we investigate the physics governing the transition between the ejection/charging regimes and characterize the ejection mode within each regime. We also discuss the implications of ejection modes and charging mechanisms in different regimes on the MS response for analytes that follow different routes to dry ion formation from the condensed phase. Importantly, the insight gained from this analysis not only provides important guidelines for understanding operation and optimal design of existing and emerging liquid-spraying-based ion sources used in mass spectrometry [17, 19-22, 24, 25], but also relevant to a number of other emerging applications such as inkjet printing $[26,27]$ and drop-on-demand manufacturing [28].

\section{Scaling Analysis}

Consider the ejection of a liquid electrolyte from an orifice with characteristic size, $l$, driven by periodic pressure forcing 
with frequency, $f$, and subjected to an external electric field of strength $E_{o}$. The ejection process is governed by the momentum equations (Navier-Stokes equations of motion) completed with the boundary conditions of surface stress balances [19]. The non-dimensionalization of the governing equations yields the following relevant time scales for the physical processes: the pressure forcing (process) time scale $\left(t_{p} \sim 1 / f\right)$, inertia time scale based on a characteristic ejection velocity $u_{o}\left(t_{u} \sim l / u_{o}\right)$, and Coulombic time scale expressed in terms of fluid density and an absolute value of the bulk charge density $q_{o}\left(t_{c} \sim \sqrt{\rho l / q_{o} E_{o}}\right)$. For the micrometer-size droplets relevant to electrospray $(l \sim 1 \mu \mathrm{m})$, the viscous and dielectric force time scales are more than an orderof-magnitude longer than the ejection process time scale, and can be neglected. Therefore, different behavior modes of electromechanical atomization are expected to occur due to interplay among the processdriving time scale (due to periodic pressure forcing), the fluid inertia time scale, and the time scale of Coulombic force interactions between the free charges in the solution. The relative magnitudes of these terms will determine behavior of a fluid upon electromechanical atomization. By forming balances among these three terms, three distinct regimes of ejection can be identified.

In the first regime, the Coulombic force is negligible, resulting in a balance between the pressure forcing and inertia terms. In this limiting regime, the electric field has little or no effect, resulting in purely mechanical ejection. That is, mechanical ejection occurs when (1) the pressure forcing and inertia time scales are of the same order, $t_{p} \sim t_{u}$, and (2) the Coulombic time scale is much longer, $t_{c} \gg t_{p}, t_{u}$. The first condition $\left(1 / f \sim l / u_{o}\right)$ provides a scale for the minimum characteristic velocity, $u_{o} \sim f l$, of the purely mechanically-driven atomization. Further, depending on the relative magnitude of the capillary (due to surface tension, $\gamma$ ) and pressure forcing time scales, expressed in terms of a relationship between the Weber $\left(W e=\rho u_{0}^{2} l / \gamma\right)$ and Strouhal $\left(S t=f l / u_{0}\right)$ numbers, either a continuous jet $\left(W e>1 / S t^{2}\right)$ or discrete droplets $\left(W e<1 / S t^{2}\right)$, are ejected upon fluid atomization [9]. Rearranging the second condition on the Coulombic time scale, the parametric region for purely mechanical ejection is determined as $q_{0} E_{o} \ll \rho l f^{2}$. This relationship isolates and compares the electric parameters (electric field and fluid ionic strength/charge density) to the mechanical parameters (orifice size and driving frequency of pressure forcing) and fluid properties (fluid density). It also demonstrates that the purely mechanical regime of ejection is not just determined by the external electric field, but by the product of the characteristic charge density and electric field strength.

In the opposite limit, the electric field dominates and drives all flow processes, resulting in steady-state electrospraying on the ejection time scale. The necessary condition for this regime is that the Coulombic time scale is much shorter than all other relevant processes, $t_{c} \ll t_{p}, t_{u}$. Here, the parametric region for pure electro- spraying is derived as, $q_{0} E_{o} \gg \rho u_{0}^{2} /$. For this condition, the determination of the appropriate characteristic velocity and length scale is less straight forward. Electrospraying can be achieved from a wide range of capillary sizes and flow rates, and occurs in a number of different regimes. A number of parametric relationships and scaling laws have been developed for spray current, minimum flow rate, electric field for the onset of spraying, and droplet/jet diameter. For example, Fernandez de la Mora and Loscertales have developed the following relationship for the minimum volumetric flow rate, $Q$, for electrospray, $Q_{\min } \sim \varepsilon_{r, g a s} \varepsilon_{o} \gamma / \rho \sigma$ [29]. The flow rate required for stable electrospraying can be used to obtain a characteristic velocity $u_{o} \sim Q / d^{2}$. However, the determination of an appropriate jet/ droplet diameter to derive the velocity scale is ambiguous. Various relationships exist in the literature for the diameter of electrospraying jet/droplets, based on empirically chosen parameters to fit experimental data [15, 29-31]. As an example, Gañán-Calvo et al. developed the following expression for an electrospraying jet diameter, $d_{j} \sim 3.78 \pi^{-2 / 3} 0.6 Q^{1 / 2}\left(\rho \varepsilon_{o} / \gamma \sigma\right)^{1 / 6}[31]$. From these relationships, an order-of-magnitude estimate for the electric field magnitude necessary for Coulombically dominated regime of ejection of an aqueous solvent containing $0.1 \%(\mathrm{vol} / \mathrm{vol})$ acetic acid $\left(\sim 50,000 \mathrm{C} / \mathrm{m}^{3}\right)$ is estimated to be $\sim 10^{7} \mathrm{~V} / \mathrm{m}$. This is in close agreement with the electric field magnitude necessary for the onset of electrospraying as developed by Smith, $E_{\text {onset }} \sim$ $\sqrt{2 \gamma \cos \theta / \varepsilon_{r, g a s} \varepsilon_{0} r_{\text {capillary }}}$ [32]. It is important to note that while this scale analysis identifies the broad regime transition from purely mechanical to coupled electromechanical atomization to pure electrospraying, it is not applicable for identifying the transition between modes within the electrospraying regime, i.e., the dripping mode [33], burst mode [16], pulsating mode [34, 35], astable mode [36], and the cone-jet mode [37].

Lastly, an important regime lies at the transition between purely mechanical ejection and electrospraying, resulting in a regime of coupled electromechanical atomization. In this case, the process, inertia, and Coulombic time scales are all of the same order, $t_{p} \sim t_{u} \sim$ $t_{c}$, and play competing roles in spray generation. This should occur when $\left(q_{o} E_{o}\right)_{\text {trans }} \sim \rho l f^{2}$. This transition between the ejection regimes is investigated using optical visualization of the ejection phenomena.

\section{Experimental Characterization}

To validate the scaling relationships for transition between the different ejection regimes and to gain insight into the spray features (ejection mode, strength, and stability) in each regime, optical imaging of ultrasonically-actuated atomization from the AMUSE ion source is performed in the presence of an external electric field. The atomization setup and electrode arrangement for locally applying an electric field has been adopted from [19, 20], and additional details can be found in the Supple- 
mentary Material, which can be found in the electronic version of this article. Stroboscopic visualization is used to gain insight into microscopic structure of the atomized spray [9]. This technique, which can be traced back to Lord Rayleigh's original work [2, 3, 38], provides still images of the periodic ejection process, "frozen" in space/time. Figure 1 displays a representative case of fluid atomization in discrete droplet mode from an array of $5 \mu \mathrm{m}$ orifices at $0.735 \mathrm{MHz}$, using both macroscale view (to show the atomization "quality") and high-spatial-resolution stroboscopic imaging with $400 \times$ magnification (to provide microscopic details of ejection from an individual orifice). From the ultrasonic atomization regime maps developed in [9], the discrete droplet ejection mode is expected with and without an applied external electric field for the operating conditions considered here (5 $\mu \mathrm{m}$ spray orifice and $0.5-1.5$ $\mathrm{MHz}$ forcing frequency, resulting in the flow rate per nozzle of $\sim 5 \mu \mathrm{L} / \mathrm{min}$ ).

Under the application of a $1 \times 10^{6} \mathrm{~V} / \mathrm{m}$ external electric field across the nozzle orifices using the external counter electrode configuration introduced in [19, 20], atomization is expected to fall into the transition regime of coupled electromechanical atomization. In this case, as shown in Figure 2, overall (macroscale) structure of

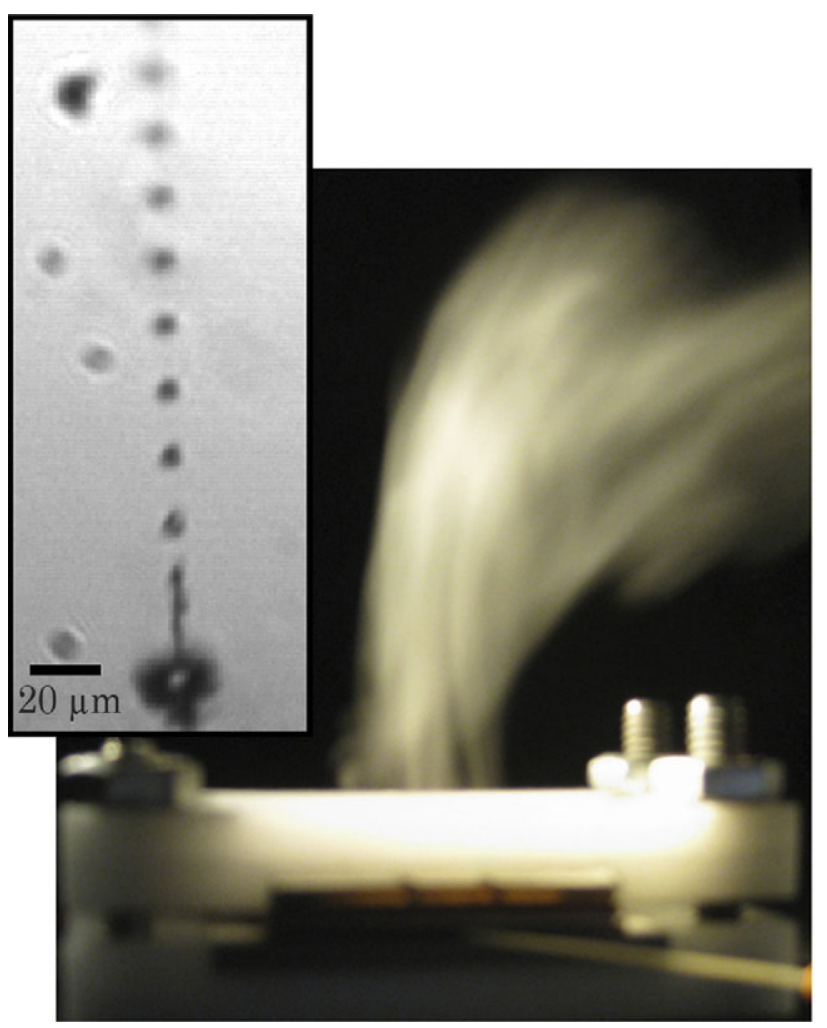

Figure 1. Representative macroscopic image of ultrasonicallygenerated spray for the purely mechanical atomization regime, i.e., when electric field strength is less that the threshold given by $q_{o} E_{o} \ll \rho l f^{2}$. Inset is a representative stroboscopic image of discrete droplet stream without the application of an electric field. These images are for an aqueous solution containing $0.1 \%$ (vol/vol) glacial acetic acid ( $\mathrm{pH} 3.25$, absolute charge $\left.\sim 50,000 \mathrm{C} / \mathrm{m}^{3}\right)$.

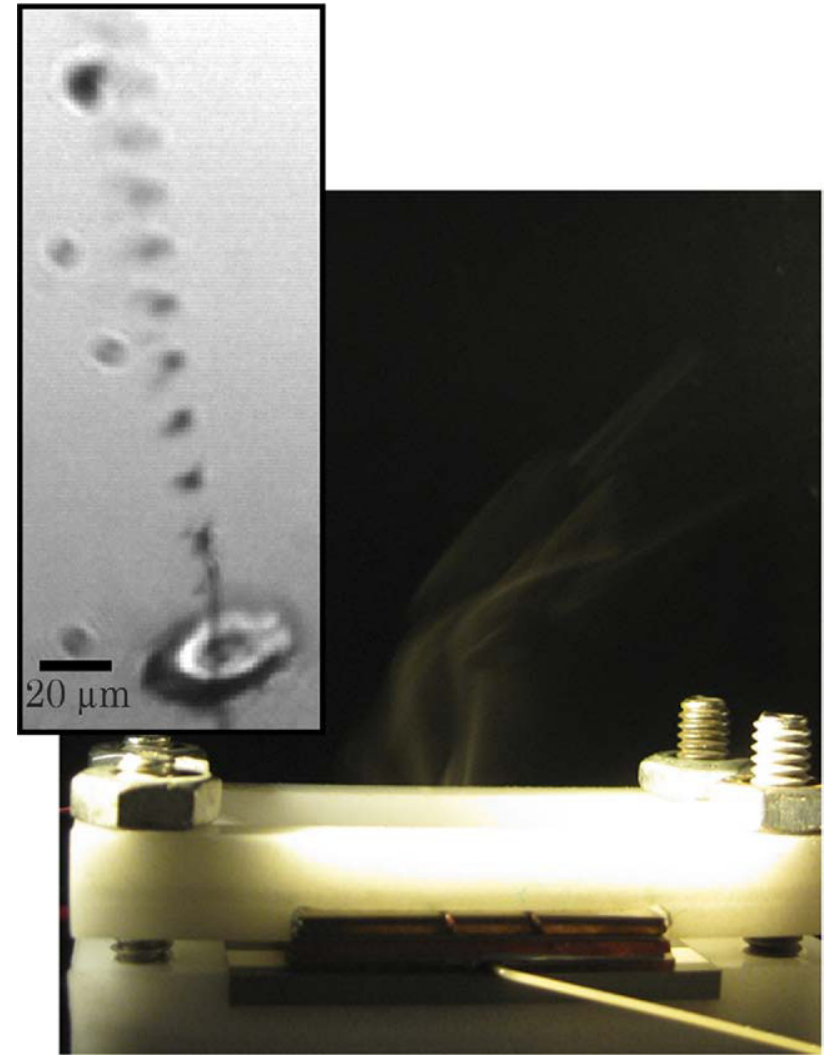

Figure 2. Representative macroscopic image of ultrasonicallygenerated spray for the coupled electromechanical atomization regime, i.e., when $\left(q_{0} E_{0}\right)_{\text {trans }} \sim \rho l f^{2}$. Inset is a representative stroboscopic image of a discrete droplet stream formed under the external electric field of $1 \times 10^{6} \mathrm{~V} / \mathrm{m}$. These images are for an aqueous solution containing $0.1 \%(\mathrm{vol} / \mathrm{vol})$ glacial acetic acid $(\mathrm{pH}$ 3.25 , absolute charge $\left.\sim 50,000 \mathrm{C} / \mathrm{m}^{3}\right)$.

the spray is greatly altered; however, the mode of ejection is largely unaffected. This regime still results in a discrete droplet mode of ejection (Figure 2 inset), however, spray atomization "quality" (macroscopic image in Figure 2) is adversely affected by distortion in the local electric field (shielding due to consecutive ejection of charged droplets) and fission of ejected droplets. That is, the ejection strength (defined as the spray height of droplet streams above the atomization orifices), ejection uniformity (number of active orifices in the atomization array), and stability during an extended period of time change drastically in this regime. Stroboscopic images of ejected droplets in the coupled electromechanical atomization regime display blurring as they move away from the nozzle array (Figure 2 inset). This is due to the strong interactions between highly charged droplets, affecting periodicity and therefore the clarity of stroboscopic images. While achieving an electric field high enough to attain electrospraying from the ultrasonic atomization setup is not realizable due to earlier onset of dielectric breakdown, the ejection of highly charged droplets approaching the Rayleigh limit [39] is possible. Once ejected, these highly charged droplets repel each other and interact with the external 
counter electrode. What are isolated streams of droplets without the application of an electric field, display a plume-like behavior at high electric fields, suggesting the importance of in-flight electrostatic repulsion. A combination of these interactions results in a significant increase in electric field magnitudes locally, which, in conjunction with droplet evaporation, promote fission of highly charged droplets upon ejection. This is why a coupled electromechanical ejection right near the atomization orifice still maintains coherent droplet streams (Figure 2 inset), typical of purely mechanical atomization, but these streams quickly deteriorate into a fissioning mist seen in a macroscopic image of the spray (Figure 2).

As alluded to in the previous discussion, the transition from purely mechanical ejection to purely electrical atomization (electrospray) occurs through a coupled electromechanical atomization regime. Thus, it would be of great interest to develop a criterion for regime transition that can be expressed as a relationship between relevant dimensionless numbers. These can be obtained from the Navier-Stokes equation of motion generalized to include electric effects [19]. Specifically, the Strouhal number, $S t=f l / u_{o}$, and a new dimensionless group, the Fenn number, $\mathrm{Fe}=q_{0} E_{0} l / \rho u_{o}^{2}$, encompass the relevant external forcing, inertia, and Coulombic interactions. From the time scale analysis, the transition from the purely mechanical to a coupled electromechanical atomization regime should occur when $t_{u} \sim$ $t_{c}$, which implies the relationship $S t \sim 1 / F^{0.25}$ for the atomization regime transition. To validate this fundamental scaling law, a series of experiments were performed for atomization from a $5 \mu \mathrm{m}$ diameter orifice at different forcing frequencies for two different ionic strength solutions under varying external electric field strength. The results are used to populate the regime map in Figure 3, identifying an onset of the coupled electromechanical atomization regime and supporting the theoretical prediction of the relationship where regime transition should occur. The specific value of the power exponent " $1 / 4$ " in the relationship between the dimensionless $S t$ and Fe numbers at the regime transition is readily recovered from the log-log plot, as shown in the inset of Figure 3.

\section{Discussion}

Characterization of electromechanical atomization regimes enables an MS practitioner to quickly gain a ready-to-use insight on the mode of ejection that can be expected for a different ion source and its performance characteristics, e.g., droplet sizes, ejection stability, maximum sample throughput, etc. This is particularly important since a set of variables relevant to device operation (liquid conductivity, electric field strength, frequency and amplitude of device operation signal, nozzle diameter, etc.) is typically large, and the relationship between dimensionless groups of parameters, such as the Strouhal and Fenn numbers, allows for a simple,

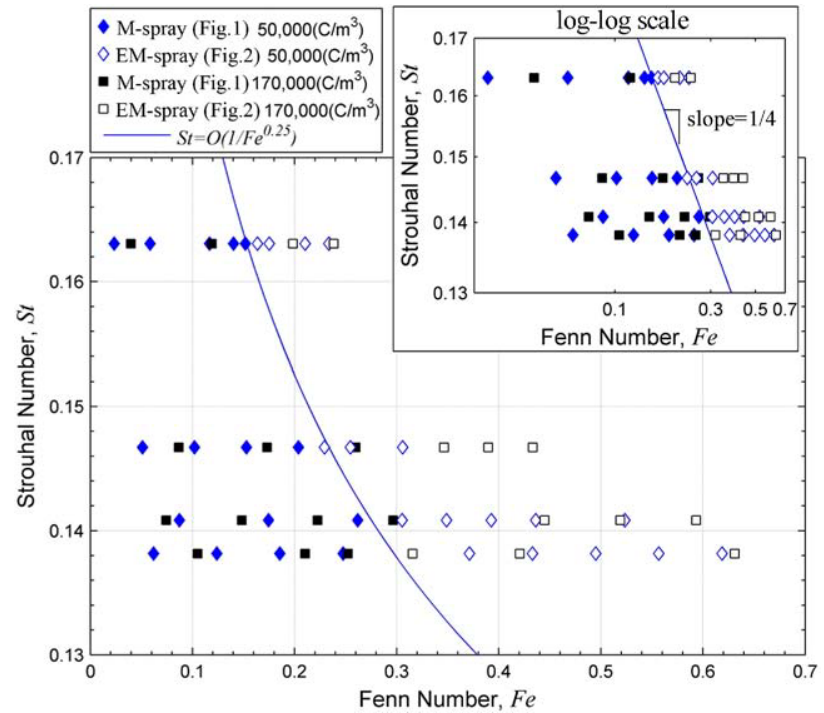

Figure 3. Regime map displaying the relationship between the Strouhal number and the Fenn number for the atomization transition from purely mechanical (abbreviated as "M-spray" as represented by Figure 1) to coupled electromechanical atomization (abbreviated as "EM-spray" as represented by Figure 2), plotted with experimental results. The inset displays the plot on a $\log$-log scale, resolving the $1 / 4$ power exponent in $\mathrm{St}$ versus $\mathrm{Fe}$ relationship.

yet general way to identify a specific ejection regime along with its implications on the analyte charging and ionization. Additionally, for a specific analyte of interest, depending on its physico-chemical properties (e.g., hydrophobicity) one may be able to define the most appropriate ejection regime in which to operate an ion source to achieve an optimal ionization efficiency based on the mechanism of dry ion formation that a given analyte is expected to follow. For example, when considering ionization of small molecules for which the ion evaporation mechanism (IEM) dominates [40], operation in the coupled electromechanical atomization regime is expected to provide the optimal mass spectrometric response. In this case, the small molecules reside on the surface of charged droplets, along with the majority of the free charge carriers, and upon charge fluctuation they are transferred into an energetically favorable gas-phase state [40-42]. In a particular case of the AMUSE ion source, in the coupled electromechanical atomization regime, the device ejects discrete droplets containing a specific amount of charge, which fission into a mist of much smaller highly charged droplets. This post-ejection induced fissioning provides an enhancement in the surface area-to-volume ratio, leading to an increase in the number of dry ions formed by the IEM. Indeed, the mass spectrometric response (signal abundance and sensitivity) for the small molecules, e.g., reserpine, has been previously shown to improve steadily across the regime transition from the purely mechanical to electromechanical atomization in the AMUSE [20]. 
For larger macromolecules or highly hydrophilic molecules, which experience ionization via the charge residue mechanism [43], operation in the coupled electromechanical atomization regime may actually be detrimental to the ionization efficiency. These molecules reside in the interior of charged droplets and require sufficient solvent evaporation and numerous Coulombic fission events before the analyte is completely desolvated and ionized by condensed residual charge carriers. In this case, improvements in signal abundance and sensitivity are unlikely to continue upon transition from purely mechanical to electromechanical atomization. It has been demonstrated that at $\sim 80 \%$ of the Rayleigh limit, highly charged droplets fission $\sim 20$ satellite droplets. These satellite droplets carry away a disproportionately large amount $(\sim 15 \%)$ of the charge and only $\sim 2 \%$ of the mass from the parent droplet [44]. Therefore, for ion sources generating relatively large droplets (e.g., AMUSE) it is quite likely that a coupled electromechanical mode of ejection will force the overcharged droplets to lose most of their excess charge during the early fission events experienced right at the ejection point and therefore leaving no sufficient residual charge for efficient ionization of a macromolecule, which remains in the parent droplet and completely desolvates further downstream closer to the MS sampling capillary. Thus, in such a case, operation of an ion source in the purely mechanical ejection regime would be beneficial to improving ionization efficiency.

\section{Conclusions}

We propose that the new dimensionless number that emerged from our analysis be named the Fenn number $\left(F e=q_{0} E_{0} l / \rho u_{0}^{2}\right)$, which is used to identify an onset of coupled electromechanical atomization as a transition between mechanically-dominated and electricallydominated regimes of atomization. The Fenn number describes the interplay between the inertia and Coulombic forces and determines the dominant mechanism driving fluid flow and atomization process. This parameter would appropriately bear the name of John Fenn, who shared the 2002 Nobel Prize in Chemistry for his invention of electrospray ionization of biomolecules. Given Fenn's fundamental contributions not only to purely electrospraying [45-47] but also ultrasonicallyassisted [24] and pneumatically-assisted [48] electrospraying, the parameter named in his honor is well suited to define the criterion for transition between purely mechanical ejection and electrospraying of electrically charged liquids. The relationship between the Strouhal and Fenn numbers $\left(S t \sim 1 / \mathrm{Fe}^{0.25}\right)$ for the regime transition in electromechanical atomization provides a valuable insight into the underlying physics and characteristics of observed ejection regimes in droplet-based ion sources. Importantly for an MS practitioner, this simple relationship allows identification of an optimal set of operating parameters, i.e., liquid conductivity, electric field strength, frequency and amplitude of the device operation signal, nozzle diameter, etc., which should lead to an improved MS response for a given analyte of interest.

\section{Acknowledgments}

The authors acknowledge that the project described was supported by grant no. 1 R21 RR021474-01A1 from the National Center for Research Resources (NCRR), a component of the National Institutes of Health $(\mathrm{NIH})$. Its contents are solely the responsibility of the authors and do not necessarily represent the official views of NCRR or NIH.

\section{Appendix A Supplementary Material}

Supplementary material associated with this article may be found in the online version at doi:10.1016/ j.jasms.2010.07.007.

\section{References}

1. Rayleigh, L. J. W. S. On the Instability of Jets. Proc. London Math. Soc. 1878, 10, 4-13.

2. Rayleigh, L. J. W. S. On the Capillary Phenomena of Jets. Proc. R. Soc. London 1879, 29, 71-97.

3. Rayleigh, L. J. W. S. Further Observations Upon Liquid Jets, in Continuation of those Recorded in the Royal Society's 'Proceedings' for March and May 1879. Proc. R. Soc. London 1882, 34, 130-145.

4. Merrington, A. C.; Richardson, E. G. The Break-Up of Liquid Jets. Proc. Phys. Soc. 1947, 59(1), 1-13.

5. Weber, C. Zum Zerfall eines Flussigkeitsstrahles [On the Decay of a Liquid Jet]. J. Appl. Math. Mech. 1931, 11(2), 136-154.

6. Suryo, R.; Basaran, O. A. Dripping of a Liquid from a Tube in the Absence of Gravity. Phys. Rev. Lett. 2006, 96(3), 034504.

7. Utada, A. S.; Fernandez-Nieves, A.; Stone, H. A.; Weitz, D. A. Dripping to Jetting Transitions in Co-Flowing Liquid Streams. Phys. Rev. Lett. 2007, 99(9), 094502.

8. Chen, A. U.; Notz, P. K.; Basaran, O. A. Computational and Experimental Analysis of Pinch-Off and Scaling. Phys. Rev. Lett. 2002, 88(17), 174501

9. Meacham, J. M.; Varady, M. J.; Degertekin, F. L.; Fedorov, A. G. Droplet Formation and Ejection from a Micromachined Ultrasonic Droplet Generator: Visualization and Scaling. Phys. Fluids 2005, 17(10), 100605100608.

10. Zeleny, J. Instability of Electrified Liquid Surfaces. Phys. Rev. 1917, 10(1), 1 .

11. Taylor, G. I. Disintegration of Water Drops in an Electric Field. Proc. $R$. Soc. London, Ser. A 1964, 280(1382), 383-397.

12. Notz, P. K.; Basaran, O. A. Dynamics of Drop Formation in an Electric Field. J. Colloid Interface Sci. 1999, 213(1), 218-237.

13. Collins, R. T.; Harris, M. T.; Basaran, O. A. Breakup of Electrified Jets. J. Fluid Mech. 2007, 588, 75-129.

14. Hohman, M. M.; Shin, M.; Rutledge, G.; Brenner, M. P. Electrospinning and Electrically Forced Jets. I. Stability Theory. Phys. Fluids 2001, 13(8), 2201-2220.

15. Collins, R. T.; Jones, J. J.; Harris, M. T.; Basaran, O. A. Electrohydrodynamic Tip Streaming and Emission of Charged Drops from Liquid Cones. Nat. Phys. 2008, 4(2), 149-154.

16. Marginean, I.; Nemes, P.; Vertes, A. Order-Chaos-Order Transitions in Electrosprays: The Electrified Dripping Faucet. Phys. Rev. Lett. 2006, 97, 064502.

17. Aderogba, S.; Meacham, J. M.; Degertekin, F. L.; Fedorov, A. G.; Fernandez, F. M. Nanoelectrospray Ion Generation for High-Throughput Mass Spectrometry Using a Micromachined Ultrasonic Ejector Array. Appl. Phys. Lett. 2005, 86(20), 203110-203113.

18. Fedorov, A. G., Degertekin,, F. L. Electrospray Systems and Methods. U.S. Patent NO. 7,208,727; 2007

19. Forbes, T. P.; Degertekin, F. L.; Fedorov, A. G. Electrohydrodynamics of Charge Separation in Droplet-Based Ion Sources with Time-Varying Electrical and Mechanical Actuation. J. Am. Soc. Mass Spectrom. 2010, 21, 501-510.

20. Forbes, T. P.; Dixon, R. B.; Muddiman, D. C.; Degertekin, F. L.; Fedorov, A. G. Characterization of Charge Separation in the Array of Micromachined UltraSonic Electrospray (AMUSE) Ion Source for Mass Spectrometry. J. Am. Soc. Mass Spectrom. 2009, 20(9), 1684-1687.

21. Hampton, C. Y.; Forbes, T. P.; Varady, M. J.; Meacham, J. M.; Fedorov, A. G.; Degertekin, F. L.; Fernandez, F. M. Analytical Performance of a Venturi-Assisted Array of Micromachined Ultrasonic Electrosprays 
Coupled to Ion Trap Mass Spectrometry for the Analysis of Peptides and Proteins. Anal. Chem. 2007, 79(21), 8154-8161.

22. Hampton, C. Y.; Silvestri, C. J.; Forbes, T. P.; Varady, M. J.; Meacham, J. M.; Fedorov, A. G.; Degertekin, F. L.; Fernández, F. M. Comparison of the Internal Energy Deposition of Venturi-Assisted Electrospray Ionization and a Venturi-Assisted Array of Micromachined UltraSonic Electrosprays (AMUSE). J. Am. Soc. Mass Spectrom. 2008, 19(9), 1320-1329.

23. Meacham, J. M.; Ejimofor, C.; Kumar, S.; Degertekin, F. L.; Fedorov, A. G. Micromachined Ultrasonic Droplet Generator Based on a Liquid Horn Structure. Rev. Sci. Instrum. 2004, 75(5), 1347-1352.

24. Banks, J. F.; Shen, S.; Whitehouse, C. M.; Fenn, J. B. Ultrasonically Assisted Electrospray Ionization for LC/MS Determination of Nucleosides from a Transfer RNA Digest. Anal. Chem. 1994, 66(3), 406-414.

25. Berggren, W. T.; Westphall, M. S.; Smith, L. M. Single-Pulse Nanoelectrospray Ionization. Anal. Chem. 2002, 74(14), 3443-3448.

26. Mutoh, M. A Study on the Drop Formation of Continuous Liquid Jet by an Electrical Method. Phys. Fluids 2002, 14(4), 1380-1388.

27. Suzuki, M.; Asano, K. A Mathematical Model of Droplet Charging in Ink-Jet Printers. J. Phys. D Appl. Phys. 1979, 12, 529-537.

28. Orme, M.; Courter, J.; Liu, Q.; Huang, C.; Smith, R. Electrostatic Charging and Deflection of Nonconventional Droplet Streams Formed from Capillary Stream Breakup. Phys. Fluids 2000, 12(9), 2224-2235.

29. Fernandez de la Mora, J.; Loscertales, I. G. The Current Emitted by Highly Conducting Taylor Cones. J. Fluid Mech. 1994, 260, 155-184.

30. Fernandez de la Mora, J.; Navascues, J.; Fernandez, F.; Rosell-Llompart, J. Generation of Submicron Monodisperse Aerosols in Electrosprays. J. Aerosol Sci. 1990, 21(special issue), S673-S676.

31. Gañán-Calvo, A. M.; Dávila, J.; Barrero, A. Current and Droplet Size in the Electrospraying of Liquids. Scaling Laws. J. Aerosol Sci. 1997, 28(2), 249-275.

32. Smith, D. P. H. The Electrohydrodynamic Atomization of Liquids. IEEE Trans. Ind. Appl. 1986, IA-22(3), 527-535.

33. Zhang, X.; Basaran, O. A. Dynamics of Drop Formation from a Capillary in the Presence of an Electric Field. J. Fluid Mech. 1996, 326, 239-263.

34. Marginean, I.; Nemes, P.; Parvin, L.; Vertes, A. How Much Charge Is There on a Pulsating Taylor Cone? Appl. Phys. Lett. 2006, 89(6), 064104064103.
35. Marginean, I.; Parvin, L.; Heffernan, L.; Vertes, A. Flexing the Electrified Meniscus: The Birth of a Jet in Electrosprays. Anal. Chem. 2004, 76(14), 4202-4207.

36. Marginean, I.; Nemes, P.; Vertes, A. Astable Regime in Electrosprays. Phys. Rev. E. 2007, 76(2), 026320.

37. Fernandez de laMora, J. The Fluid Dynamics of Taylor Cones. Annu. Rev. Fluid Mech. 2007, 39, 217-243.

38. Rayleigh, L. J. W. S. Some Applications of Photography. Nature 1891, 44, $249-254$.

39. Rayleigh, L. J. W. S. On the Equilibrium of Liquid Conducting Masses Charged with Electricity. Philos. Mag. 1882, 14, 184-186.

40. Iribarne, J. V.; Thomson, B. A. On the Evaporation of Small Ions from Charged Droplets. J. Chem. Phys. 1976, 64(6), 2287-2294.

41. Bakhoum, S. F. W.; Agnes, G. R. Study of Chemistry in Droplets with Net Charge before and after Coulomb Explosion: Ion-Induced Nucleation in Solution and Implications for Ion Production in an Electrospray. Anal. Chem. 2005, 77(10), 3189-3197.

42. Cole, R. B. Some Tenets Pertaining to Electrospray Ionization Mass Spectrometry. J. Mass Spectrom. 2000, 35(7), 763-772.

43. Dole, M.; Mack, L. L.; Hines, R. L.; Mobley, R. C.; Ferguson, L. D.; Alice, M. B. Molecular Beams of Macro-Ions. J. Chem. Phys. 1968, 49(5), 2240-2249.

44. Kebarle, P.; Tang, L. From Ions in Solution to Ions in the Gas Phase-the Mechanism of Electrospray Mass Spectrometry. Anal. Chem. 1993, 65(22), 972A-986A

45. Fenn, J. B. Ion Formation from Charged Droplets: Roles of Geometry, Energy, and Time. J. Am. Soc. Mass Spectrom. 1993, 4(7), 524-535.

46. Fenn, J. B.; Mann, M.; Meng, C. K.; Wong, S. F.; Whitehouse, C. M. Electrospray Ionization for Mass Spectrometry of Large Biomolecules. Science 1989, 246(4926), 64-71.

47. Whitehouse, C. M. Dreyer, R. N.; Yamashita, M. Fenn, J. B. Electrospray Interface for Liquid Chromatographs and Mass Spectrometers. Anal. Chem. 1985, 57(3), 675-679.

48. Yamashita, M.; Fenn, J. B. Electrospray Ion Source. Another Variation on the Free-Jet Theme. J. Phys. Chem. 1984, 88(20), 4451-4459. 